\title{
Virtual Fluoroscopy: Safe Zones for Pelvic Screw Fixations
}

\author{
B. König ${ }^{1}$, U. Stöckle ${ }^{1}$, M. Sati ${ }^{2}$, L.P. Nolte ${ }^{2}$, N.P. Haas ${ }^{1}$ \\ ${ }^{1}$ Unfall- und Wiederherstellungschirurgie \\ Humboldt Universität zu Berlin, Charité, Campus Virchow Klinikum, Augustenburgerplatz 1, \\ 13353 Berlin, Germany \\ E-mail: benjamin.koenig@charite.de \\ ${ }^{2}$ M.E. Müller Institute for Biomechanics, Murtenstr.35, P.O. Box 30, CH- 3010 Bern, \\ Switzerland
}

\begin{abstract}
Hypothesis: Percutaneous pelvic screw fixations are technically demanding.

Purpose of this prospective study: To define appropriate fluoroscopy projections for the safe placement of SI screws, anterior column screw, posterior column screw and iliac screw using fluoroscopy based navigation.

Material \& Methods: A conventional C-arm $\left(\mathrm{Ziehm}{ }^{\circledR}\right)$ and two different navigation systems were used (Medivision SurgiGate ${ }^{\circledR}$ and Sofamore Danek Stealth Station ${ }^{\circledR}$ ). In 6 pelvic models and 4 cadaver pelves three to four projections per screw position were defined and a total of 84 screws were inserted. Evaluation of accuracy was performed with X-ray, CT and dissection.
\end{abstract}

Results: The following fluoroscopy projections proved to be appropriate:

SI srews: $\quad$ Lateral, lateral $20^{\circ}$ a.p., inlet, outlet

Ant. Column: $\quad$ Inlet $30^{\circ}$ ipsilateral, obturator outlet ipsilateral, obturator outlet contralateral, obturator

Post. Column: Obturator outlet ipsilateral, obturator outlet contralateral, outlet $20^{\circ}$ contralateral

Iliac screw: $\quad$ Inlet $30^{\circ}$ ipsilateral, obturator ipsilateral, obturator contralateral

The average fluoroscopy time was 6 seconds/ screw in pelvic models and 86 seconds/ screw in cadaver pelves.

In the first series with 60 screws in the pelvic models, 51 screws were inserted correctly. In five cases there was minor displacement without perforating the cortex, in four cases the cortex was perforated. In the cadaver study 19 of 24 screws were placed correctly, two were satisfactory and three were not satisfactory (one SI, ant. Column, post. Column each).

Conclusion: With a remarkable low fluoroscopy time of 6 seconds/ screw in pelvic models and 86 seconds/ procedure in cadaver pelves $83 \%$ of all pelvic screws were inserted perfectly. With further improvements in fluoroscopic image quality the method of fluoroscopy based navigation will be very helpful not only for pelvic screw fixations but for many applications in Traumatology.

\section{Introduction}

Percutaneous screw fixations of the pelvis are technically ambitious because of the complex anatomy of the pelvis. The screws are inserted under fluoroscopic control or 
CT-guidance to stabilize pelvic ring injuries or acetabular fractures. Procedures under fluoroscopic control need rather long fluoroscopy times for the correct positioning. The drill can only be controlled in one projection at a time and at least three different projections are necessary. It is not uncommon that several drilling procedures are necessary with repetitive fluoroscopic controls in different projections. This can result in fluoroscopy times of ten minutes and more per screw.

For CT-based navigation the matching procedure can be difficult as the bony structures are covered. For each reduction maneuver the acquisition of a new data set is necessary. Therefore either the CT has to be located in the operating room or surgery has to take place in the CT. This can cause logistic problems.

The new method of fluoroscopy based navigation has the advantage that a fluoroscope is available in any orthopedic OR. The image acquisition in the needed projections is a quick procedure. A maximum of 4 different projections can be displayed on the navigation screen with direction and length of a referenced drill. According to the display on the screen the drill is inserted.

Before the clinical use of this promising new method it was necessary to perform an experimental study with pelvic models to define appropriate fluoroscopy projections for the most common percutaneous screws. In the next step the precision of screws which were inserted under fluoroscopy based navigation was to be evaluated with Xray, CT and dissection of the pelvic models. The second part of this study was to verify the practicability of these predefined projections in human cadavers for pelvic screw fixation.

\section{Material and Methods}

A conventional $\mathrm{C}$-arm (Ziehm ${ }^{\circledR}$ ) was used for the first part of the study with the fluoroscopy based navigation module SurgiGate $\AA$ of Medivision $\AA$ and for screw insertion in human cadavers with the Stealth Station ${ }^{\circledR}$ navigation system of Sofamore Danek ${ }^{\circledR}$. Optoelectronic markers are mounted on instruments, the dynamic reference base and the C-arm. Three (SurgiGate $\AA$ ) or two (Stealth Station ${ }^{\circledR}$ ) infrared cameras assess the position of the LED's in the three dimensional space.

On pelvic models (Synthes $\left.{ }^{\circledR}\right)$ appropriate fluoroscopy projections were defined for these pelvic screws:

\section{- Sacroiliacal screw in S1}

- Sacroiliacal screw in S2

- Anterior column

- Posterior column

- Supraacetabular iliac screw

The fluoroscopic images had to display the osseous borders of the screw pathways and important anatomic structures like the acetabulum. With respect to standard projections they had to be reproducible.

In a second trial the five screws were inserted on each side using fluoroscopy based navigation. Cannulated screws with a diameter of $7.0 \mathrm{~mm}$ were used. The precision of 60 screws in pelvic models was assessed by X-ray, CT and dissection. Fluoroscopy time was documented. 
Afterwards in four cadavers twenty-four screws were inserted: six SI- screws in S1 and six in S2, four anterior column, one posterior column and two iliac screws. Screw position and accuracy of the system were analyzed by means of stored system settings, postoperative radiological and CT- scans as well as dissection of the models. Fluoroscopy time and radiation dose was registered.

\section{Results}

\subsection{Definition of Appropriate Fluoroscopy Projections}

Combinations of the standard pelvic projections inlet / outlet and iliac / obturator view were defined as appropriate projections.

- SI screws: $\quad$ Inlet, outlet, lateral, lateral $20^{\circ}$ a.p.

- Iliac screw: Inlet $30^{\circ}$ ipsilateral, obturator ipsilateral, obturator contralateral

- Anterior column: Inlet $30^{\circ}$ ipsilateral, obturator outlet ipsilateral, obturator outlet contralateral, obturator

- Posterior column: Obturator outlet ipsilateral, obturator outlet contralateral, outlet $20^{\circ}$ contralateral

\subsection{Precision of the Screws}

All the 60 screws in pelvic models were inserted according to the defined views. The average fluoroscopy time per screw was 6 seconds.

51 of those 60 screws $(85 \%)$ were placed correctly. The postoperative X-ray correlated with the screen displays and in CT as well as in dissection there was no perforation of the cortex. In five cases (two SI and three anterior column screws) there were deviations from the screen display with acceptable screw position without joint or cortex laceration.

Two SI screws in pelvic models were misplaced with cortex perforation respectively intraforaminal placement of the screw tip. Additionally two anterior column screws were malpositioned, one with cranial perforation of the pubic ramus and one with intraarticular placement of 2 threads.

In the cadaver study 19 of 24 screws (79\%) where placed correctly. Two screws where located satisfactory without perforation of cortex and three screws perforated the cortex. Those were one SI, anterior column and posterior column each.

The used projections for pelvic screws fixation proved to be good. Accuracy of the navigation system was sufficient. The number of required pictures of each projection considerable varied. On average 86 seconds of radiation time was needed per procedure. 


\section{Discussion}

Because of the complex pelvic anatomy percutaneous screw fixations are technically difficult. Yet cannulated screws are used under fluoroscopic control. As different projections have to be controlled separately a repetitive correction of the guide wire can be necessary to achieve the correct position. This can result in fluoroscopy times of 10 minutes per screw [10].

Procedures under CT control offer high precision of the inserted screws especially when performed with CT based navigation $[1,2,5]$. However, in case of reduction maneuvers a new data set has to be acquired after each manipulation. Therefore either a CT is needed in the operating room or surgery has to be performed in the CT. This can cause logistic problems.

Fluoroscopy based navigation is a new method in which the fluoroscope, the patient and the instruments are referenced with optoelectronic markers. Infrared cameras assess the actual position in the three dimensional space [3,4]. There is the advantage that a fluoroscope already is available in the orthopedic operating room and is in frequent use. The necessary fluoroscopy projections are acquired only at the beginning of the procedure to be displayed on the screen simultaneously. As a prerequisite the $\mathrm{C}$-arm projections have to be defined exactly for each screw position to enable a correct screw placement. In this study appropriate $\mathrm{C}$-arm projections were defined for five common screw positions in the pelvis. Using these views a reduction of fluoroscopy time to 6 seconds per screw was possible in pelvic models. In the cadaver study the needed fluoroscopy time ( 86 seconds per procedure) was higher than in the previous series. Of course this extreme low fluoroscopy time in artificial pelvis is only possible in the experimental setup. But it is promising for the clinical use.

In this experimental study a correct placement of the screws could be achieved in 83 $\%$. This equals the results of the conventional technique under fluoroscopic control. The misplacements in artificial pelves were in five cases minor deviations without perforation of the cortex and in four cases with perforation of the cortex for pelvic models. In the cadaver study 19 of 24 screws were placed correctly, two were satisfactory and three were not satisfactory. The reasons for the deviations from the planned position were insufficient image quality and technical failures.

A good image quality of the fluoroscope in the defined views is a mandatory prerequisite for a safe and precise placement of the drill. In six of nine misplacements in pelvic models and all three misplacements in cadavers the visualization was impaired by already inserted screws or was misinterpreted. The placement of ten percutaneous screws into one pelvis is an extreme situation with less clinical relevance. However, further improvement of the image quality of the fluoroscope is necessary as two or three percutaneous screws are realistic.

Technical failures were mainly caused by deviations of thin drills from the planned pathway. This is also known from the clinical use of the guide wire for the $7.0 \mathrm{~mm}$ screw.

The defined C-arm projections proved to be appropriate for safe placement of percutaneous pelvic screws using fluoroscopy based navigation. With remarkable low fluoroscopy times a high precision of the screws could be achieved. With further 
improvements in fluoroscopic image quality the method of fluoroscopy based navigation will be very helpful not only for pelvic screw fixations but for many applications in Traumatology.

\section{References}

1. Ebrahim NA et al: Percutaneous computed tomography-guided stabilization of posterior pelvic fractures. Clin Orthop 1994 Oct (307): 222-8

2. Gay SB et al: Percutaneous screw fixation of acetabular fractures with CT-guidance: preliminary results of a new technique. Am J Roentgenol 1992 Apr; 158(4): 819-22

3. Hamadeh A et al: Automated 3-dimensional computed tomographic and fluoroscopic image registration. Comput Aided Surg 1999; 4(2): 65-76

4. Hofstetter R, Slomczykowski M, Sati M, Nolte P: Fluoroscopy as an imaging means for computed assisted surgical navigation. Comput Aided Surg 1999; 4(2): 65-76

5. Jacob AL et al: Posterior pelvic ring fractures: closed reduction and percutaneous CTguided sacroiliac screw fixation. Cardiovasc Intervent Radiol 1997 Jul-Aug; 20(4): 285-94

6. Parker PJ et al: Percutaneous fluoroscopic screw fixation of acetabular fractures. Injury 1997 Nov-Dec; 28(9-10): 597-600

7. Routt ML Jr et al: Early results of percutaneous ilisacral screws placed with the patient in the supine position. J Orthop Trauma 1995 Jun; 9(3): 207-14

8. Routt ML Jr, Simonian PT: Closed reduction and percutaneous skeletal fixation of sacral fractures. Clin Orthop 1996 Aug; (329):121-8

9. Shuler TE et al: Percutaneous ilisacral screw fixation; early treatment for unstable posterior pelvic ring disruptions. J Trauma 1995 Mar; 38(3):453-8

10. Starr AJ et al: Percutaneous fixation of the columns of the acetabulum: a new technique. J Orthop Trauma 1998 Jan; 12(1):51-8 